\title{
IDENTIFICATION OF A POLYNOMIAL IN NONSEPARATED BOUNDARY CONDITIONS IN THE CASE OF A MULTIPLE ZERO EIGENVALUE
}

\author{
A.M. AKHTYAMOV, R.R. KUMUSHBAEV
}

\begin{abstract}
In the work we discuss the problem of recovering the coefficients of a polynomial in spectral problems with nonseparated boundary conditions by one multiple zero eigenvalue and $n$ nonzero eigenvalues. A uniqueness theorem is proved.
\end{abstract}

Keywords: eigenvalues, boundary conditions, characteristic determinant.

Mathematics Subject Classification: 35L75, 65A18, 34K29

\section{INTRODUCTION}

In solving applied problems of mathematical physics, there appear spectral problem involving polynomially a spectral parameter in boundary conditions [1]-[4], as well as problems with an operator in boundary conditions [5]. In the associated inverse problems, by known spectra one has to recover the unknown coefficients in equations and boundary conditions [6]-[13]. In [14], a polynomial is separated boundary conditions was recovered by a finite set of different eigenvalues. In [15], a polynomial of degree $m$ in nonseparated boundary conditions was recovered by $m+1$ different eigenvalues. However, the information on multiplicities of the eigenvalues was not employed in [15]. In the present paper we make use a multiplicity of zero eigenvalue. To recover the polynomial in this case we employ less number of eigenvalues $(<m)$.

\section{Formulation of the PROBlem}

We consider the following spectral problem:

$$
\begin{gathered}
y^{\prime \prime}+p_{1}(x, \lambda) y^{\prime}+p_{2}(x, \lambda) y=0, \\
U_{i}(y)=a_{i 1}(\lambda) y^{\prime}(0)+a_{i 2}(\lambda) y(0)+a_{i 3}(\lambda) y^{\prime}(1)+a_{i 4}(\lambda) y(1)=0,
\end{gathered}
$$

where $\lambda$ is a spectral parameter; $i=1,2 ; x \in[0,1] ; p_{1}(x, \lambda), p_{2}(x, \lambda)$ are continuously differentiable functions w.r.t. $x$ and $\lambda ; a_{i j}(i=1,2, j=1,2,3,4)$ are continuously differentiable functions w.r.t. $\lambda$ and

$$
\sum_{j=1}^{4}\left|a_{i j}(\lambda)\right| \neq 0, \quad \text { as } i=1,2 \text { and for each } \lambda \text {. }
$$

A.M. Akhtyamov, R.R. Kumushbaev, Identification of the Polynomial in nonseparated BOUNDARY CONDITIONS FOR MULTIPLE ZERO EIGENVALUE CASE.

(C) Akhtyamov A.M., Kumushbaev R.R. 2015.

The work is supported by the Council on grants of the President of Russian Federation (grant NSh1096.2014.1), RFBR (grants 14-01-97010-r_povolzhe_a, 15-01-01095_a), the Ministery of Education and Science of the Republic of Kazakhstan (grants MON RK 2217/GFZ, MON RK 2989/GFZ).

Submitted August 24, 2014. 
In the present paper we solve an inverse problem. Suppose that one of the functions $a_{2 j}(\lambda)$ $(j=1,2,3,4)$, which we denote by $a_{2 p}(\lambda)$, is a polynomial:

$$
a_{2 p}(\lambda)=\sum_{s=0}^{m} a_{2 p s} \lambda^{s}
$$

We know $n+1$ eigenvalues $\lambda_{0}, \lambda_{1}, \ldots, \lambda_{n}$ of problem (1), (2). One of them $\lambda_{0}=0$ has the multiplicity $r_{0}$, at that, $m=n+r_{0}-1$ is the degree of polynomial $a_{2 p}(\lambda)$. We need to recover polynomial $a_{2 p}(\lambda)$.

\section{UNIQUENESS THEOREM}

We denote by $y_{1}(x, \lambda)$ and $y_{2}(x, \lambda)$ linearly independent solutions to differential equation (1) satisfying the conditions

$$
y_{1}(0, \lambda)=1, \quad y_{1}^{\prime}(0, \lambda)=0, \quad y_{2}(0, \lambda)=0, \quad y_{2}^{\prime}(0, \lambda)=1 .
$$

Eigenvalues $\lambda_{k}$ are roots of the characteristic determinant [16]

$$
\Delta(\lambda)=\left|\begin{array}{ll}
U_{1}\left(y_{1}\right) & U_{1}\left(y_{2}\right) \\
U_{2}\left(y_{1}\right) & U_{2}\left(y_{2}\right)
\end{array}\right|=\sum_{j=1}^{4} a_{2 j}(\lambda) A_{2 j}(\lambda)
$$

where

$$
\begin{aligned}
& A_{21}(\lambda)=a_{12}(\lambda)+a_{13}(\lambda) y_{1}^{\prime}(1, \lambda)+a_{14}(\lambda) y_{1}(1, \lambda) \\
& A_{22}(\lambda)=-a_{11}(\lambda)-a_{13}(\lambda) y_{2}^{\prime}(1, \lambda)-a_{14}(\lambda) y_{2}(1, \lambda) \\
& A_{23}(\lambda)=a_{12}(\lambda) y_{2}^{\prime}(1, \lambda)+a_{14}(\lambda) W(1, \lambda)-a_{11}(\lambda) y_{1}^{\prime}(1, \lambda) \\
& A_{24}(\lambda)=a_{12}(\lambda) y_{2}(1, \lambda)-a_{11}(\lambda) y_{1}(1, \lambda)-a_{13}(\lambda) W(1, \lambda) \\
& W(1, \lambda)=y_{1}(1, \lambda) y_{2}^{\prime}(1, \lambda)-y_{1}^{\prime}(1, \lambda) y_{2}(1, \lambda), \text { as } k=0,1, \ldots, n .
\end{aligned}
$$

If $p_{1}(x, \lambda) \equiv 0$, then by (4) and by the Liouville formula for the Wronskian [17, Ch. V, Subsect. 17.1] we obtain that $W\left(1, \lambda_{k}\right)=1$.

Function $A_{2 p}(\lambda)$ introduced in (5) with $p$ chosen above is expressed via known coefficients $a_{2 j}$ and known functions $y_{1}(x, \lambda)$ and $y_{2}(x, \lambda)$.

Theorem. Polynomial $a_{2 p}(\lambda)$ of degree $m$ in boundary condition (2) is uniquely recovered by zero eigenvalue $\lambda_{0}=0$ of multiplicity $r_{0}$ and by $n=m-r_{0}+1$ non-zero mutually different eigenvalues $\lambda_{1}, \lambda_{2}, \ldots, \lambda_{n}$ if $A_{2 p}\left(\lambda_{k}\right) \neq 0, k=0,1, \ldots, n$

Proof. Let us show that as $A_{2 p}\left(\lambda_{k}\right) \neq 0$, polynomial $a_{2 p}(\lambda)$ is uniquely recovered, while for $A_{2 p}\left(\lambda_{k}\right)=0$ the unique recovering is impossible.

Let $A_{2 p}\left(\lambda_{k}\right) \neq 0$. It follows from identities $\Delta\left(\lambda_{k}\right)=0$ and (4) that

$$
a_{2 p}\left(\lambda_{k}\right)=-\sum_{j=1, j \neq p}^{4} a_{2 j}\left(\lambda_{k}\right) \frac{A_{2 j}\left(\lambda_{k}\right)}{A_{2 p}\left(\lambda_{k}\right)}, \quad k=0,1, \ldots, n .
$$

Substituting the known eigenvalues into (4), we obtain a system of algebraic equations for unknown coefficients $a_{2 p s}$ :

$$
a_{2 p 0}+a_{2 p 1} \lambda_{k}^{1}+\ldots+a_{2 p m} \lambda_{k}^{m}=-\sum_{j=1, j \neq p}^{4} a_{2 j}\left(\lambda_{k}\right) \frac{A_{2 j}\left(\lambda_{k}\right)}{A_{2 p}\left(\lambda_{k}\right)},
$$

where $k=0,1, \ldots, n$. 
System of linear algebraic equations (7) has $m+1$ unknowns and $n+1=\left(m-r_{0}+2\right)$ equations. It is impossible to identify uniquely the coefficients of the polynomial since the amount of unknowns in this system is greater than the number of equations. However, by the assumption, $\lambda_{0}=0$ has multiplicity $r_{0}$.

The multiplicity of the root follows that

$$
\left\{\begin{array}{l}
\Delta\left(\lambda_{0}\right)=0 \\
\Delta^{\prime}\left(\lambda_{0}\right)=0 \\
\cdots \\
\Delta^{\left(r_{0}-1\right)}\left(\lambda_{0}\right)=0 \\
\Delta^{\left(r_{0}\right)}\left(\lambda_{0}\right) \neq 0
\end{array}\right.
$$

By (5) and (8) we obtain

$$
\left\{\begin{array}{l}
\Delta\left(\lambda_{0}\right)=\sum_{j=1}^{4} a_{2 j}\left(\lambda_{0}\right) A_{2 j}\left(\lambda_{0}\right), \\
\Delta^{\prime}\left(\lambda_{0}\right)=\sum_{j=1}^{4}\left(a_{2 j}^{\prime}\left(\lambda_{0}\right) A_{2 j}\left(\lambda_{0}\right)+a_{2 j}\left(\lambda_{0}\right) A_{2 j}^{\prime}\left(\lambda_{0}\right)\right), \\
\ldots \\
\Delta^{\left(r_{0}-1\right)}\left(\lambda_{0}\right)=\sum_{j=1}^{4}\left(C_{r_{0}-1}^{0} a_{2 j}^{\left(r_{0}-1\right)}\left(\lambda_{0}\right) A_{2 j}\left(\lambda_{0}\right)+\ldots+C_{r_{0}-1}^{r_{0}-1} a_{2 j}\left(\lambda_{0}\right) A_{2 j}^{\left(r_{0}-1\right)}\right) .
\end{array}\right.
$$

Employing (9) and eigenvalue $\lambda_{0}=0$, we find the first $r_{0}$ coefficients of polynomial $a_{2 p s}(\lambda)$ by means of the recurrent relations

$$
\begin{aligned}
a_{2 p i}= & -\frac{\sum_{j=1, j \neq p}^{4}\left(C_{i}^{0} a_{2 j}^{(i)}(0) A_{2 j}(0)+C_{i}^{1} a_{2 j}^{(i-1)}(0) A_{2 j}^{\prime}(0)+\cdots+C_{i}^{i} a_{2 j}(0) A_{2 j}^{(i)}(0)\right)}{A_{2 p}(0)} \\
& -\frac{\left(C_{i}^{1} a_{2, p, i-1} A_{2 p}^{\prime}(0)+C_{i}^{2} a_{2, p, i-2} A_{2 p}^{\prime \prime}(0)+\cdots+C_{i}^{i} a_{2 p 0} A_{2 j}^{(i)}(0)\right)}{A_{2 p}(0)},
\end{aligned}
$$

where $i=0,1, \ldots, r_{0}-1$.

Therefore, the desired polynomial reads as

$$
a_{2 p}(\lambda)=a_{2 p 0}+a_{2 p 1} \lambda+\ldots+a_{2 p r_{0}-1} \lambda^{r_{0}-1}+\ldots+a_{2 p m} \lambda^{m},
$$

where $a_{2 p 0}, \ldots, a_{2 p r_{0}-1}$ are determined by means of recurrent relations (10), while other coefficients $a_{2 p r_{0}}, \ldots, a_{2 p m}$ are unknown. Let us find them by other $n=\left(m-r_{0}+1\right)$ known mutually different eigenvalues $\lambda_{1}, \ldots, \lambda_{n}$ of problem (1), (2).

We denote the known part of polynomial $a_{2 p}(\lambda)$ as

$$
V(\lambda):=a_{2 p 0}+a_{2 p 1} \lambda+\ldots+a_{2 p r_{0}-1} \lambda^{\left(r_{0}-1\right)} .
$$

Then system of equations (7) becomes

$$
a_{2 p r_{0}} \lambda_{k}^{r_{0}}+\ldots+a_{2 p m} \lambda_{k}^{m}=-\sum_{j=1, j \neq p}^{4} a_{2 j}\left(\lambda_{k}\right) \frac{A_{2 j}\left(\lambda_{k}\right)}{A_{2 p}\left(\lambda_{k}\right)}-V\left(\lambda_{k}\right),
$$


where $A_{2 p}\left(\lambda_{k}\right) \neq 0$, and $k=1,2, \ldots, m-r_{0}+1$. By the assumption, eigenvalues $\lambda_{1}, \lambda_{2}, \ldots, \lambda_{m-r_{0}+1}$ are mutually different and are non-zero. Then we divide all the equations in system (11) by $\lambda_{k}^{r_{0}}, k=\overline{1, m-r_{0}+1}$, to obtain

$$
a_{2 p r_{0}}+\ldots+a_{2 p m} \lambda_{k}^{m-r_{0}}=-\sum_{j=1, j \neq p}^{4} a_{2 j}\left(\lambda_{k}\right) \frac{A_{2 j}\left(\lambda_{k}\right)}{A_{2 p}\left(\lambda_{k}\right) \lambda_{k}^{r_{0}}}-\frac{V\left(\lambda_{k}\right)}{\lambda_{k}^{r_{0}}} .
$$

The determinant of system (12) w.r.t. unknowns $a_{2 p s}, s=r_{0}, \ldots, m$, is the Vandermonde determinant

$$
\Delta=\left|\begin{array}{cccc}
1 & \lambda_{1} & \ldots & \lambda_{1}^{m} \\
1 & \lambda_{2} & \ldots & \lambda_{2}^{m} \\
\vdots & \vdots & & \vdots \\
1 & \lambda_{n} & \ldots & \lambda_{n}^{m}
\end{array}\right|=\left(\lambda_{n}-\lambda_{n-1}\right) \ldots\left(\lambda_{n}-\lambda_{1}\right) \ldots\left(\lambda_{2}-\lambda_{1}\right) \neq 0
$$

Hence, system of equations (12) has the unique solution determined, for example, by Cramer's formulae:

$$
a_{2 p r_{0}}=\frac{\Delta_{1}}{\Delta}, \ldots, a_{2 p m}=\frac{\Delta_{n}}{\Delta},
$$

where the determinants $\Delta_{i}(i=1, \ldots, n)$ are obtained from determinant $\Delta$ by replacing $i$ th column by the column of the right hand sides in system of equations (12). The proof for the case $A_{2 p}\left(\lambda_{k}\right) \neq 0$ is complete. The solution to the inverse problem is given by means of formulae (10) and (13).

If polynomial $A_{2 p}(\lambda)$ vanishes at points $\lambda=\lambda_{k}$, it follows from representation (5) that the identity $\Delta\left(\lambda_{k}\right)=0$ is possible for each $a_{2 p}\left(\lambda_{k}\right)$. This is in the case $A_{2 p}\left(\lambda_{k}\right)=0$ polynomial $a_{2 p}\left(\lambda_{k}\right)$ is recovered non-uniquely. The proof is complete.

\section{EXAMPLES}

Example 1. We consider the following problem

$$
\begin{aligned}
& -y^{\prime \prime}=\lambda^{2} y, \\
& y^{\prime}(0)+y(1)=0, \\
& y^{\prime}(1)-a_{24}(\lambda) y(1)=0,
\end{aligned}
$$

where $a_{24}(\lambda)=a_{240}+a_{241} \lambda+a_{242} \lambda^{2}+a_{243} \lambda^{3}+a_{244} \lambda^{4}$. We need to recover the coefficients of polynomial $a_{24}(\lambda)$ by three eigenvalues. Eigenvalue $\lambda_{0}=0$ has multiplicity three and we know two other eigenvalues $\lambda_{1}=\pi, \lambda_{1}=2 \pi$. The characteristic determinant of the problem reads as:

$$
\Delta(\lambda)=1+\lambda \sin (\lambda)-a_{24}(\lambda) \cos \lambda .
$$

Since $p=4$, we have $A_{24}=-\cos \lambda$ and $A_{24}(0)=1 \neq 0$. Employing equation (10) and eigenvalue $\lambda_{0}=0$, we find first three coefficients of polynomial $a_{24}(\lambda)$ :

$$
a_{240}=1, \quad a_{241}=0, \quad a_{242}=\frac{3}{2} .
$$

Then our polynomial becomes

$$
a_{24}(\lambda)=1+\frac{3}{2} \lambda^{2}+a_{243} \lambda^{3}+a_{244} \lambda^{4} .
$$


Coefficients $a_{243}$ and $a_{244}$ can be recovered by eigenvalues $\lambda_{1}=\pi$ and $\lambda=2 \pi$ by means of formula (13):

It follows that

$$
a_{243}=\frac{\Delta_{2}}{\Delta}=-\frac{4}{\pi^{3}}-\frac{9}{4 \pi}, \quad a_{244}=\frac{\Delta_{1}}{\Delta}=\frac{2}{\pi^{4}}+\frac{3}{4 \pi^{2}}
$$

$$
a_{24}(\lambda)=1+\frac{3}{2} \lambda^{2}-\left(\frac{4}{\pi^{3}}+\frac{9}{4 \pi}\right) \lambda^{3}+\left(\frac{2}{\pi^{4}}+\frac{3}{4 \pi^{2}}\right) \lambda^{4} .
$$

Example 2. The characteristic determinant for the spectral problem

$$
\begin{aligned}
& -y^{\prime \prime}=\lambda^{2} y, \\
& y^{\prime}(0)-y^{\prime}(1)=0, \\
& y(1)-a_{22} y(0)=0,
\end{aligned}
$$

reads as:

$$
\Delta(\lambda)=\left(1+a_{22}\right)(\cos \lambda-1) .
$$

The unique recovering of coefficient $a_{22}$ by eigenvalue $\lambda=0$ of this problem is impossible, since, condition $A_{22}(0) \neq 0$ fails. Indeed, $A_{22}(0)=-a_{11}-a_{13} y_{2}^{\prime}(0)-a_{14} y_{2}(0)=-1+1$. $\cos (0)-0 \cdot \sin (0)=0$.

\section{BIBLIOGRAPHY}

1. A.A. Shkalikov. Boundary value problems for ordinary differential equations with a parameter in the boundary conditions // Trudy Sem. im I.G. Petrovskogo. 9, 190-229 (1983). (in Russian).

2. N.Yu. Kapustin, E.I. Moiseev. Spectral problems with the spectral parameter in the boundary condition // Differ. Uravn. 33:1, 115-119 (1997). [Differ. Equat. 33:1, 116-120 (1997).]

3. A.M. Akhtyamov. Calculation of the coefficients of expansions in derivative chains of a spectral problem // Matem. Zametki. 51:6, 137-139 (1992). [Math. Notes. 51:6, 618-619 (1992).]

4. A.M. Akhtyamov. On coefficients of eigenfunction expansions for boundary-value problems with parameter in boundary conditions // Matem. Zametki. 75:4, 493-506 (2004). [Math. Notes. 75:34, 462-474 (2004).]

5. S.S. Mirzoev, A.R. Aliev, L.A. Rustamova. On the boundary value problem with the operator in boundary conditions for the operator-differential equation of second order with discontinuous coefficients // Zh. Mat. Fiz. Anal. Geom. 9:2, 207-226 (2013).

6. I.M. Nabiev, A.Sh. Shukurov. Properties of the spectrum and uniqueness of reconstruction of SturmLiouville operator with a spectral parameter in the boundary condition // Proc. Inst. Math. Mech. Nat. Acad. Sci. Azerbaijan. 40, 332-341 (2014).

7. Kh.R. Mamedov, F. Cetinkaya. Inverse problem for a class of Sturm-Liouville operator with spectral parameter in boundary condition // Bound. Value Probl. id 2013:183, 16pp (2013).

8. N.B. Kerimov, Kh.R. Mamedov. On a boundary value problem with a spectral parameter in the boundary conditions // Sibir. Matem. Zhurn. 40:2, 281-290 (1999). [Sib. Mat. Zh. 40:2, 325-335 (1999).]

9. E.S. Panakhov, H. Koyunbakan, Ic. Unal. Reconstruction formula for the potential function of Sturm-Liouville problem with eigenparameter boundary condition // Inverse Prob. Sci. Eng. 18:1, 173-180 (2010).

10. M.V. Chugunova. Inverse spectral problem for the Sturm-Liouville operator with eigenvalue parameter dependent boundary conditions in book "Operator Theory, System Theory and Related Topics". Oper. Theory: Adv. Appl. Birkhäuser, Basel. 123, 187-194 (2001).

11. C. van der Mee, V. N. Pivovarchik. A Sturm-Liouville inverse spectral problem with boundary conditions depending on the spectral parameter // Funkts. Anal. Pril. 36:4, 74-77 (2002). [Funct. Anal. Appl. 36:4, 315-317 (2002).] 
12. V.A. Sadovnichii, Ya.T. Sultanaev, A.M. Akhtyamov. Inverse problem for an operator pencil with nonseparated boundary conditions // Dokl. Akad. Nauk. 425:1, 31-33 (2009). [Dokl. Math. 79:2, 169-171 (2009).]

13. G. Freiling, V. Yurko. Inverse problems for Sturm-Liouville equations with boundary conditions polynomially dependent on the spectral parameter // Inverse Probl. 26:5, id 055003, 17pp (2010).

14. A.M. Akhtyamov. Determination of the boundary condition of the basis of a finite set of eigenvalues // Differ. Uravn. 35:8, 1127-1128 (1999). [Differ. Equat. 35:8, 1141-1143 (1999).]

15. A.M. Akhtyamov, R.R. Kumushbaev. Identification of a polynomial in nonsplitting boundary conditions // Differ. Uravn. 48:11, 1549-1552 (2012). [Differ. Equat. 48:11, 1527-1530 (2012).]

16. M.A. Najmark. Linear differential operators. Nauka, Moscow (1969). (in Russian).

17. E. Kamke. Differentialgleichungen. I: Gewöhnliche Differentialgleichungen. Akademische Verlagsgesellschaft Geest und Portig K.G., Leipzig (1969).

Akhtyamov Azamat Mukhtarovich,

Bashkir State University,

Zaki Validi str., 32,

450076, Ufa, Russia

Institute of Mechanics,

Ufa Scientific Center, Russian Academy of Sciences,

Oktyabrya av., 71,

450054, Ufa, Russia

E-mail: AkhtyamovAM@mail.ru

Kumushbaev Rustem Raimanovich,

Bashkir State University,

Zaki Validi str., 32,

450076, Ufa, Russia

E-mail: KumushbaevR@gmail.com 